\title{
DESEMPEÑO PROFESIONAL DE LOS ORIENTADORES EN LOS INSTITUTOS DE EDUCACIÓN SECUNDARIA DE SALAMANCA. CREACIÓN DE UN INSTRUMENTO DE VALORACIÓN Y AUTOVALORACIÓN
}

\author{
PROFESSIONAL PERFORMANCE OF SECONDARY SCHOOL \\ COUNSELLORS IN SALAMANCA. DEVELOPMENT OF AN EVALUATION \\ AND SELF-EVALUATION INSTRUMENT
}

\author{
Francisco Royo* \\ Universidad de Salamanca
}

\begin{abstract}
RESUMEN
El trabajo que se presenta en este artículo pretende dar luz sobre el actual desempeño profesional del orientador en los institutos de educación secundaria, asimismo sirve para la creación de un instrumento de medida válido para la valoración y autovaloración de las funciones del orientador. Para ello hemos analizado las respuestas dadas por los orientadores de los institutos de secundaria de la provincia de Salamanca, tanto en las entrevistas personales mantenidas con ellos, como en la respuesta a los diferentes instrumentos de valoración. Los resultados sugieren dos factores principales que influyen en el desempeño del orientador, por un lado el apoyo del equipo directivo y por otro el modelo de trabajo tanto de los profesores del centro como del propio orientador. Por último se presenta un modelo para futuras líneas de investigación, así como un anexo en el que los orientadores manifiestan sus preocupaciones personales y profesionales.
\end{abstract}

Palabras Clave: psicología educativa, orientación educativa, orientación vocacional, educación secundaria, enseñanza, aprendizaje, tutoría, tutor, atención a la diversidad

\begin{abstract}
The work reported in this paper aim to enlighten about the present professional performance of secondary school counsellors. At the same time, it has been created an evaluation and self-evaluation instrument of counsellor's tasks. Answers to personal interviews and to evaluation instruments from Secondary School Salamanca educational counsellors has been analysed. Results suggest that two prin-
\end{abstract}

* Francisco Royo Mas es Becario de investigación en el Dpto. de Psicología Evolutiva y de la Educación. Facultad de Psicología de la Universidad de Salamanca. 
cipal factors are influencing in performance, in one hand the support of management team and in the other hand the counsellor and teachers team model of working. In the end we present two appendix, a model of future research lines about these subjects and some personal and professional worries of counsellors.

Key Words: educational psychology, educational guidance, vocational counselling, secondary school, teaching, learning, tutorial classes, class teacher, attention to diversity

\section{Introducción}

Estas páginas están dedicadas al análisis del desempeño profesional del orientador («profesor de psicología y pedagogía») en los Institutos de Educación Secundaria públicos de la provincia de Salamanca.

El objetivo que perseguimos es estudiar el grado de desarrollo de las tres funciones básicas que según la legislación competen a los Orientadores de secundaria: Apoyo al Plan de Acción Tutorial (PAT), Apoyo al Plan de orientación Académica y Profesional (POAP) y Apoyo al Proceso de Enseñanza-Aprendizaje (PEA).

Se asume normalmente que estas tres funciones deben desarrollarse adoptando una posición colaborativa con los profesores, lo que supone que el desarrollo de esta labor puede conceptualizarse como un proceso de resolución conjunta de problemas $\mathrm{y}$, por tanto, establecer alguna forma de distribución de la responsabilidad en los cuatro momentos claves del proceso: la definición del problema, la meta a la que quieren llegar, los pasos para llegar a esa meta y cómo se va a saber si se alcanzan o no los objetivos propuestos.

Éste es un proceso que se autorregula a sí mismo y que puede ir variando sobre la marcha siempre y cuando queden claros los objetivos y lo que se va a hacer, y que la supervisión se realice adecuadamente. En todo caso, una evaluación final rigurosa y consensuada por todos, sirve, a modo de investigación en la acción, para regular la próxima práctica.

Otro elemento destacado en este proceso que no debe olvidarse es el apoyo institucional, ya que según se desprende de la legislación y de las manifestaciones de los orientadores, es éste un aspecto clave requerido para un buen desarrollo de la labor orientadora.

La cuestión es que aunque estas ideas parece que cuentan con un amplio consenso entre los profesionales como modelo a seguir, parece necesario estudiar lo que realmente ocurre en la vida profesional de los orientadores.

Con esta idea en mente hemos entrevistado a 24 orientadores de los 25 Institutos públicos de Educación Secundaria de la provincia de Salamanca, y para ello hemos desarrollado un instrumento para evaluar el desempeño de la práctica orientadora según un modelo colaborativo de las tres funciones.

De esta manera los objetivos más concretos del trabajo son:

a) justificar el instrumento de evaluación creado

b) mostrar las evidencias que se pueden reunir en un estudio exploratorio en el que se evaluó con ese instrumento al $50 \%$ de toda la muestra.

Como puede verse, los objetivos del estudio se dirigen, por un lado, a proponer un instrumento de medida para analizar la práctica de los profesionales de la orientación. Y por 
otro lado, y como resultado del primer objetivo, exponer los datos que hemos extraído al emplear dicho instrumento en un estudio exploratorio.

La organización de estas páginas es la que sigue: en primer lugar, presentamos el instrumento de medida como eje central del estudio, junto a un breve comentario de otros dos instrumentos informativos creados para apoyar a éste, posteriormente se analizan los datos extraídos con dichos instrumentos $\mathrm{y}$, finalmente, exponemos las conclusiones que nos ha sugerido el estudio detallado de los datos y su comparación con otros estudios precedentes relacionados con el tema tratado.

\section{El instrumento de evaluación}

El primer paso para la elaboración del instrumento fue entrevistar al $50 \%$ de la muestra a través de una entrevista abierta (ver ANEXO I) a la vez que rellenaban las cuestiones de un registro con información diversa (ver ANEXO II).

Para la elaboración de la entrevista y el registro nos hemos basado en diferentes fuentes bibliográficas. Por un lado están las referencias de autores relevantes en el estudio de la orientación y la intervención psicopedagógica como Isabel Solé (1998), Elena Martín (1999), Mara Selvini (1986), Jesús Alonso Tapia (1995), Eulalia Bassedas (1991), Emilio Sánchez (1995), etc. También se consultaron los documentos legislativos sobre educación relacionados con el tema que nos ocupa (LOGSE, Reales Decretos, Órdenes y Circulares).

Tras analizar las respuestas obtenidas mediante esta primera aproximación se inició la elaboración del instrumento de evaluación (ver ANEXOS 3, 4 y 5) a los que hemos llamado «Esquemas de trabajo», que son los siguientes:

(I): Apoyo al PLAN DE ACCIÓN TUTORIAL (PAT)

(II): Apoyo al PLAN DE ORIENTACIÓN ACADÉMICA Y PROFESIONAL (POAP)

\section{(III): Apoyo al PROCESO DE ENSEÑANZA-APRENDIZAJE (PEA)}

Para operativizar este proceso, hemos subdividido los esquemas I y II en cuatro partes conectadas entre sí que reflejan un modelo de trabajo colaborativo. Su estructura queda de la siguiente manera:

\section{$\left.1^{\circ}\right)$ Planificación}

\section{$2^{\circ}$ ) Actuación}

\section{$3^{\circ}$ ) Supervisión}

\section{$\left.4^{0}\right)$ Evaluación.}

La primera se corresponde con la delimitación del problema o asunto y con la fijación de las metas a alcanzar, la segunda con los pasos a seguir, y la tercera y cuarta con el modo en que los participantes van a decidir si se va por buen camino o no y si al final se ha cumplido lo previsto o no. En el caso del esquema de trabajo correspondiente al Proceso de Enseñanza y Aprendizaje (PEA), optamos por una división centrada más en las tareas que en los procesos debido a la multiplicidad de aquéllas y a la imposibilidad, por cuestiones de espacio, de una subdivisión más profunda (Anexo 5). 
Por último, antes de pasar al análisis de los datos, citamos las fuentes legislativas en las que nos hemos apoyado para elaborar los instrumentos que son tres. Por un lado la "Circular de la Dirección General de Renovación Pedagógica del 29 de abril de 1996 sobre el Plan de Actividades de los Departamentos de Orientación» que nos indica las tres funciones principales en las que debe participar el orientador ${ }^{1}$.

Y por otro, el Artículo 42 del «Real Decreto 83/1996, de 26 de enero, por el que se aprueba el Reglamento Orgánico de los Institutos de Educación Secundaria», y el punto 1 (profesorado de la especialidad de Psicología y Pedagogía) de la "Resolución de 29 de abril de 1996, de la Dirección General de Centros Escolares, sobre organización de los Departamentos de Orientación en Institutos de Educación Secundaria», que nos informan de las funciones asignadas al Departamento de Orientación en general y al orientador en particular.

\section{Análisis de los datos}

La muestra en la que basamos el estudio son la práctica totalidad de los orientadores de los Institutos de Educación Secundaria Públicos de la provincia de Salamanca, es decir, 24 de los 25 que están en activo, y ha sido realizada entre junio de 1999 y mayo de 2000.

Todos los instrumentos fueron aplicados y rellenados personalmente por el primer autor del trabajo en presencia del orientador y en el $80 \%$ de los casos en su instituto correspondiente.

El registro fue cumplimentado por los 24 componentes de la muestra (100\%), la entrevista por catorce de ellos (58\% de la muestra) y los esquemas de trabajo por once (46\%).

En lo que sigue, analizaremos los resultados obtenidos al aplicar cada uno de los esquemas. Así, y respecto del primero de ellos, el PLAN DE ACCIÓN TUTORIAL (PAT), y en concreto al primer apartado, la Planificación, nos interesan fundamentalmente dos variables:

a) Constatar cuál es el grado en el que se colabora en el diseño del plan.

b) Determinar el grado de apoyo institucional.

Ambos aspectos pueden ser considerados como los más relevantes para esta primera parte de la labor orientadora, al fin y al cabo no debe olvidarse su naturaleza colaborativa.

En referencia a la primera cuestión se recogen evidencias de varios indicadores, que son los ítems que preguntan por las aportaciones de los tutores y profesores al documento del Plan de Acción Tutorial en los diversos momentos del proceso, es decir, desde la elaboración hasta la presentación final al Claustro, pasando por la presentación a los tutores y a la Comisión de Coordinación Pedagógica.

1. «1. Plan de Actividades de los Departamentos de Orientación.

El cumplimiento de las funciones que el articulo 42 del Reglamento Orgánico de los Institutos de Educación Secundaria adjudica al Departamento de Orientación requiere la intervención de los profesionales que lo componen en tres grandes ámbitos interrelacionados: el apoyo al proceso de enseñanza y aprendizaje, la orientación académica y profesional, y la acción tutorial.» 
Los datos reunidos indican que los tutores realizan aportaciones en un $36 \%$ de los casos, es decir, que alrededor de un $64 \%$ de los orientadores señalaron que la elaboración del documento final fue responsabilidad suya. Las aportaciones de la CCP se dieron en un $27 \%$ de los casos y el Claustro aportó en un 9\% del total. Esto puede ser interpretado como que el grado de participación de los profesores es muy escaso en esta primera fase.

Respecto al apoyo institucional, en dos casos (18\%) el PAT fue presentado por el director al Claustro y en ningún caso contó el orientador con la colaboración de algún miembro del equipo directivo para su presentación a la Comisión de Coordinación Pedagógica.

Podemos resumir los resultados de la Planificación del PAT como un proceso en el que el orientador es el principal protagonista, ya que lo elabora con poca ayuda, él mismo lo presenta a los distintos órganos (tutores, Comisión de Coordinación Pedagógica y Claustro) de los que recibe escasas aportaciones y por último queda aprobado sin demasiada discusión. Quizás por todo ello y, como algunos orientadores manifestaron, es más bien aceptado que consensuado.

En lo que se refiere a la parte de las Actividades desplegadas en la hora de tutoría nos interesa saber:

a) El grado de colaboración de los tutores con los orientadores.

b) El tipo y variedad de actividades implementadas.

La información referente a la primera cuestión la recogemos directamente del ítem que pregunta por el tipo de peticiones que les hacen los tutores a los orientadores y el tipo de colaboración profesional que les demandan. Con respecto a esto, se puede ver una cierta unanimidad en los centros por parte de los tutores en dos cuestiones, una a la hora de pedir material didáctico a los orientadores (100\%), y otra la petición de impartir un determinado tipo de tutorías, más específicamente aquellas relacionadas con las salidas formativas y profesionales al término de la ESO y del Bachillerato; estas peticiones se dieron en un $90 \%$ de los casos. Por otra parte, una pregunta del cuestionario que sirve como indicador de colaboración real, es la petición de asesoramiento por parte de los tutores a los orientadores sobre cómo implementar los materiales solicitados, de la cual recogemos que en la mitad de los centros se da un porcentaje elevado de solicitud de asesoramiento.

A la luz de estos datos destacar dos hechos, primero la unanimidad con la que les es requerido a los orientadores material para las tutorías, y segundo, el que se dé un cierto predominio de tareas que no son propias del orientador, como es la de impartir tutorías, sobre otro tipo de tarea más propio de sus funciones como es el asesorar sobre cómo implementar los materiales suministrados. Estos datos se pueden interpretar, en la línea de lo visto anteriormente, como otro indicio de que la labor de orientadores y tutores hasta esta parte del proceso, sin dejar de estar conectadas, no implica una estrecha colaboración sino más bien un trabajo individual en el que ambos requieren del otro para cuestiones puntuales.

La segunda cuestión que planteábamos en esta parte del proceso era la referente al tipo y variedad de actividades que finalmente se implementan, porque va a reflejar cómo se llevan a la realidad los planes propuestos en un principio. La información sobre este aspecto la obtenemos del ítem que pregunta directamente por el tipo de actividades que se han llevado a cabo. Lo que podemos ver es que el $100 \%$ de la muestra lleva a cabo las llamadas «obligatorias»: la recepción de los alumnos, elección de delegado, inserción grupal y preparación de las evaluaciones; pero en cuanto al resto de actividades se observa una gran variabi- 
lidad entre los diferentes centros, tanto en lo referente al tipo de actividades como al número total de ellas que se realizan. En todo caso predominan las relacionadas con las educación para la salud y la prevención de ciertos riesgos como son los de la droga, alcohol y tabaco ( 7 centros). Otras actividades son: técnicas de estudio (4), educación sexual (3), transversales (2), anorexia (2), SIDA (2), planificación y hábitos de estudio (2), sociogramas (1), relaciones familiares (1), razonamiento abstracto (1), autoestima (1), integración grupal (1), solidaridad (1), habilidades creativas (1), evaluación de la tutoría (1), habilidades de relación social (1).

El tercer paso del proceso, la Supervisión, lo consideramos como un aspecto fundamental para desplegar un auténtico trabajo riguroso que no se quede simplemente en una declaración de intenciones sino que, como un buen sistema que se autorregula a sí mismo, el seguimiento de las tutorías debe contar con un espacio y un tiempo para la reflexión sobre lo hecho y la planificación de las nuevas actuaciones, y para ello es necesaria la colaboración no sólo de orientador y tutores sino también de jefatura de estudios, que es la responsable de la coordinación tanto del PAT como del POAP. Por eso nos interesa conocer información sobre variables similares a las del momento de la planificación, es decir:

a) Constatar cuál es el grado en el que se colabora durante la implementación del plan.

b) Determinar el grado de apoyo institucional.

Los indicadores que nos hablan del primer aspecto son los referidos a la decisión de reunirse, la frecuencia de las reuniones, y qué se supervisa en esas reuniones. Vemos que en dos casos (18\%) la decisión de reunirse para la supervisión está consensuada por todos, en cuatro casos (36\%) la decisión es de jefatura de estudios, en un caso deciden jefatura y orientador y en cuatro (36\%) no hay reunión de seguimiento.

El segundo indicador es la frecuencia de las reuniones, la cual debería ser semanal, y en ese sentido obtenemos cuatro respuestas (36\%), en un caso es quincenal, en otro bimensual, hay dos casos en los que la reunión es trimestral, y como hemos dicho, en cuatro casos no hay reunión. Como último indicador, a la pregunta de ¿qué se supervisa? encontramos que de los siete centros en los que se realiza supervisión, en tres de ellos se dedica exclusivamente a planificar la siguiente actividad, a diferencia de los otros cuatro que además supervisan las actividades desarrolladas esa semana.

Se puede destacar de entre estos datos que en bastantes casos hay una reunión de supervisión semanal, lo cual, sin significar directamente una garantía total de que ésta se realice adecuadamente, sí que implica una primera aproximación a esta función de una forma constante y correcta, tal como indican los documentos legislativos, ya que significa el reunirse todos los profesionales implicados en la acción tutorial durante todas las semanas para revisar lo que se ha hecho hasta ese momento y preparar las siguientes actuaciones. En los casos en que la reunión de supervisión es quincenal, mensual o incluso trimestral, se puede pensar que ésta no obtendrá la profundidad y el nivel de colaboración obtenido en las semanales.

Llaman la atención los casos en los que no se realiza reunión de seguimiento, y que puede ser un indicador parcial de una baja predisposición hacia las tareas de orientación, en todo caso no deja mucho espacio para el trabajo colaborativo.

Respecto a la segunda variable, los indicadores que nos hablan del apoyo institucional son los ítems que preguntan por las personas que asisten a las reuniones de supervisión y quiénes presiden dichas reuniones. Lo que obtenemos es que de los 7 casos en los que hay 
reunión, en cuatro de ellos (57\%) participa el jefe de estudios, mientras que en tres de ellos (43\%) no lo hace. Si sumamos los cuatro casos en los que no hay reunión de supervisión más los tres casos en los que habiendo supervisión, jefatura de estudios no participa, nos encontramos con que en el $64 \%$ de la muestra no hay apoyo institucional durante las reuniones de supervisión de la acción tutorial. Ante esta evidencia se puede pensar que el orientador queda en algunos centros desasistido en un momento clave, debido a que jefatura de estudios, que es el órgano encargado de coordinar el desarrollo de la acción tutorial mediante diferentes acciones, como convocar reuniones semanales, presidirlas, dar parte de las asistencias, etc., no lleva a la práctica dichas acciones, y esto refleja en buena manera el sentir del equipo directivo hacia las labores de orientación,

Por último, una breve reseña sobre la Evaluación para decir que ésta se realiza en todos los centros durante el mes de junio, y las aportaciones realizadas, en su caso, por tutores, alumnos y jefatura de estudios, sirven para mejorar el documento del curso siguiente. Esta evaluación es la aportación de tutores y demás agentes evaluadores, que el orientador utiliza para la confección del Plan de Acción Tutorial del curso siguiente ya que todos los orientadores manifestaron tener en cuenta las opiniones que profesores y alumnos expresaron en dichas evaluaciones finales.

Los datos obtenidos en el $2^{\circ}$ esquema, el PLAN DE ORIENTACIÓN ACADÉMICA Y PROFESIONAL (POAP), son muy similares a los del PAT, puesto que la mayoría de los tutores incluyen las actividades del POAP como parte del PAT, tanto tomando prestadas las horas de tutoría para implementarlas, como realizando su seguimiento a la vez que el del PAT.

Quizás como dato más interesante destacamos la implementación mayoritaria por los tutores, con la colaboración de los orientadores, de tres tipos de actividades: «Información sobre las rutas académicas», «Información sobre salidas laborales» y «Toma de decisiones»; y en la mitad de los casos se llevaron a la práctica dos más: «Visitas a empresas» y «Programas de informática».

El tercer esquema, el apoyo al PROCESO DE ENSEÑANZA Y APRENDIZAJE, en el que se refleja una de las áreas de intervención más significativas de la figura del orientador, no lo hemos subdividido de la misma forma que los anteriores (Anexo V), debido, como ya comentamos anteriormente, a la amplia y variada gama de funciones que se incluyen en este apartado y la consiguiente imposibilidad por razones de estructura y de espacio para acotarlo de la misma manera que los anteriores apartados sin perder la coherencia del estudio. Por eso en esta tercera función que vamos a analizar, la cantidad de apartados diferentes nos hace fijar la vista más en las diversas tareas que en los procesos, pero queremos llamar la atención una vez más sobre la importancia que estos tienen cuando el modelo desde el que se trabaja es el del asesoramiento colaborativo.

La variable en la que nos fijamos principalmente en este apartado es el grado y forma de colaboración entre diferentes profesionales a la hora de llevar a la práctica el apoyo al proceso de enseñanza-aprendizaje. Los indicadores que nos hablan sobre esto son principalmente los subapartados de la tarea «Refuerzo Educativo», y los referidos a los diferentes tipos de «Adaptaciones Curriculares». Con respecto al primero, los datos reflejan que existe trabajo en equipo de una forma generalizada, si bien éste se lleva a cabo bajo una gran diversidad tanto en lo referente a los integrantes del equipo como a los momentos en los que colaboran conjuntamente los diferentes profesionales. Como ejemplo citamos el subaparta- 
do «supervisión» en el que se dan combinaciones de todo tipo: orientador solo, profesor de área, PT y tutor (1), orientador, tutor y PT (1), orientador, tutor y profesor de área (1), orientador y PT (1), Orientador, PT y jefe de estudios (1), orientador y profesor de área (1), PT y profesor de área (1), etc. (recordemos que en algunos Dptos. no hay PT). La misma disparidad podemos ver con respecto a la intervención y la evaluación en este apartado.

Por otra parte, en el indicador «Adaptaciones Curriculares» y «Atención a ANEEs», observamos también diversidad de combinaciones de profesionales tanto en la intervención como en la supervisión y evaluación final, y sólo en la evaluación psicopedagógica hay coincidencia, al ser realizada ésta en todos los casos por el orientador.

Para finalizar el análisis de los esquemas, queremos mencionar un hecho que puede servirnos de ayuda a la hora de tratar de explicar el funcionamiento de los orientadores, y es que hemos apreciado que las funciones que tienen asignadas son muy variadas y numerosas, ya que además de las tres principales que han centrado el estudio, en la práctica se llevan a cabo otras muchas más como p. ej.: asesoramiento (Comisión de Coordinación Pedagógica, Claustro, alumnos, padres), docencia (6-9 horas), participación en reuniones de evaluación, organización y preparación de reuniones de padres, contactos con los EOEPs del sector, etc. Ante este largo listado de funciones se puede pensar que en ocasiones resulte imposible realizarlas con la profundidad y profesionalidad que requiere la labor de orientación educativa, tanto individual como grupal, en especial en aquellos centros en los que el número de alumnos está en torno a los mil.

Para complementar los datos de los esquemas vamos a repasar brevemente algunos resultados interesantes vertidos en el registro y la entrevista.

Una primera ojeada a los datos de los «registros» nos permiten deducir de nuevo la existencia de una GRAN VARIABILIDAD en aspectos esenciales del desempeño profesional del orientador. Sirva como ejemplo el $2^{\circ}$ ítem: «la experiencia docente y orientadora»; el $3^{\circ}$ : «formación previa»; y el $4^{\circ}$ : «componentes de los Dptos. de Orientación». La diversidad de la «experiencia» es tal que va desde dos años como orientador y ninguno como profesor, hasta 11 años como orientador y 20 como profesor, pasando por una multiplicidad de combinaciones sin ninguna coincidencia entre los 24 orientadores de la muestra. La misma variabilidad observamos con respecto a la formación previa, en la que si bien predomina la carrera de Pedagogía junto a otra licenciatura, podemos ver también licenciados en Psicología o en Pedagogía únicamente, en Psicología y Pedagogía, en Pedagogía y Magisterio, etc. También apreciamos gran diversidad en cuanto a los componentes de los Dptos. de Orientación, predominando el tándem orientador más tres profesores pertenecientes a los ámbitos (socio-lingüístico, científico-tecnológico, área práctica), también hay bastantes formaciones de orientador, tres ámbitos y profesor de Pedagogía Terapéutica (PT), pasando por el dúo de orientador y PT, e incluso orientador únicamente.

Con respecto a las preguntas referentes a la relación de trabajo con los otros profesionales del centro también se puede apreciar gran disparidad de opiniones. P. ej. las obtenidas en el ítem 18 que está relacionado con un modelo de trabajo de asesoramiento colaborativo, los resultados demuestran, una vez más, una gran diversidad y a su vez una baja identificación con el modelo propuesto. Por último, veamos dos ítems que nos servirán en el apartado de las conclusiones para apoyar los datos obtenidos en los esquemas de trabajo, ya que la opinión que tienen unos profesionales de otros y lo que esperan de ellos influye en el desempeño final (Tablas 1 y 2). 
TABLA 1: Ítem 18. La percepción que

tienen los demás profesionales del centro sobre lo que es un orientador es:

\begin{tabular}{|l|c|c|}
\hline CORRECTA* $^{*}$ & 11 & $46 \%$ \\
\hline INCORRECTA & 7 & $29 \%$ \\
\hline DE LAS DOS & 4 & $17 \%$ \\
\hline NO LO SABE & 2 & $8 \%$ \\
\hline
\end{tabular}

* Los que respondieron así, añadieron que se había dado un proceso que iba desde una percepción incorrecta de la figura del orientador y una actitud de indiferencia, hacia una percepción correcta y de colaboración.

TABLA 2: Ítem 19.a y 19.b. ¿Qué actitud hay en los centros escolares hacia el orientador?

\begin{tabular}{|l|c|c|l|c|c|}
\hline \multicolumn{3}{|c|}{ Ítem: 19.a } & \multicolumn{4}{c|}{ Ítem: 19.b } \\
\hline BUENA & $\mathbf{1 5}$ & $\mathbf{( 6 2 , 5 \% )}$ & $\begin{array}{l}\text { DE } \\
\text { COLABORACIÓN* }\end{array}$ & $\mathbf{1 2}$ & $\mathbf{( 5 0 \% )}$ \\
\hline MALA & $\mathbf{3}$ & $\mathbf{( 1 2 , 5 \% )}$ & DE INDIFERENCIA & $\mathbf{6}$ & $\mathbf{( 2 5 \% )}$ \\
\hline DE LAS DOS & $\mathbf{5}$ & $\mathbf{( 2 1 \% )}$ & DE AMBAS & $\mathbf{6}$ & $\mathbf{( 2 5 \% )}$ \\
\hline NO LO SABE & $\mathbf{1}$ & $\mathbf{( 4 \% )}$ & & $\mathbf{2 4}$ & $\mathbf{1 0 0}$ \\
\hline & $\mathbf{2 4}$ & $\mathbf{1 0 0}$ & \multicolumn{3}{|c}{} \\
\cline { 1 - 5 } & & &
\end{tabular}

De la «entrevista» queremos resaltar un comentario vertido por prácticamente todos los orientadores: «la COORDINACIÓN CON EL EQUIPO DIRECTIVO del centro es fundamental para un buen desarrollo de las labores de orientación». La fundamentación legal para esta suposición la encontramos en la «Resolución de 29 de abril de 1996, de la Dirección General de Centros Escolares, sobre organización de los Departamentos de Orientación en Institutos de Educación Secundaria»².

\section{Conclusiones y propuesta de trabajo}

Las principales conclusiones a las que llegamos tras el análisis de los datos obtenidos, debido a su repercusión en el desempeño profesional cotidiano de los orientadores son dos. Primeramente, y en referencia a la cuestión del apoyo institucional, si nuestros indicadores

2. Primera. De acuerdo con lo establecido en el artículo 33 del Reglamento Orgánico de los Institutos de Educación Secundaria, las actuaciones del Dpto. de Orientación deberán llevarse a cabo bajo la coordinación del Jefe de Estudios, especialmente cuando tengan alguna incidencia en la organización y funcionamiento del Instituto o supongan la participación de Profesores de diferentes departamentos. 
son válidos para detectar este aspecto, hemos visto que el equipo directivo apoya las labores de orientación educativa en algunos de los centros, pero en el resto se muestra escaso.

Las consecuencias que esto tiene para los orientadores son muy profundas puesto que el Dpto. de Orientación, al no ser departamento didáctico, se encuentra en cierta manera desasistido administrativamente, y es a través del equipo directivo, y en particular de jefatura de estudios, desde donde debe recibir un respaldo que avale sus actuaciones de orientación educativa, actuaciones, que por otro lado, están consideradas como uno de los factores de calidad del sistema educativo junto a otras que competen a profesores, equipo directivo, etc. ${ }^{3}$

Si tenemos en cuenta que una de las manifestaciones más habituales de los orientadores, por no decir unánime, es que el apoyo del equipo directivo es una pieza clave para el trabajo del orientador, este factor estaría incidiendo negativamente en el desempeño cotidiano de las labores de orientación en aquellos centros en los que los orientadores no reciben, o en su caso, no perciben, un apoyo institucional claro.

En segundo lugar, hay que hacer referencia a un aspecto fundamental para el desempeño profesional de los orientadores como es el modelo de trabajo que se utiliza en la práctica diaria, es decir, el tipo de relación profesional que se establece con los demás agentes educativos. En nuestro actual sistema educativo se asume que este debe ser el de asesoramiento colaborativo, que podemos resumir como una colaboración activa de los diferentes profesionales implicados en la resolución de cada problema, y que debe extenderse a lo largo de todo el proceso de trabajo, es decir, en la delimitación del problema o asunto a tratar, en el reparto de tareas durante la actuación, en la supervisión de esa actuación y en la evaluación final. Según los datos obtenidos, hay una buena disposición hacia la colaboración entre los distintos profesionales que trabajan en los centros, pero en algunos casos esta queda relegada a aspectos puntuales o para temas en los que es indispensable para dar salida a la burocracia prescriptiva, es decir, sin llegar a fraguar de una manera clara un trabajo colaborativo entre orientadores y el resto de agentes educativos. En los casos de Dptos. de Orientación insuficientemente dotados se llega a un ejercicio prácticamente individualista de las tareas de orientación.

Cuando no existe un apoyo institucional claro al orientador ni trabajo colaborativo, las tareas orientadoras en los centros pueden quedar sujetas a la solución particular que cada orientador proponga según su experiencia y el tipo de centro en el que trabaja, pero estará lejos de lograrse una unificación de criterios entre los profesionales de la orientación educativa a la hora del desempeño final. Por eso, si consideramos como factores fundamentales para el desempeño de los orientadores tanto el apoyo institucional como el modelo de trabajo, y éstos en ocasiones no se cumplen en la medida que sería de desear, podemos pensar que otros factores comenzarán a ejercer peso junto a los dos mencionados (ver tabla 3 ).

Una tercera conclusión que consideramos relevante destacar, extraída fundamentalmente de los datos del tercer esquema (PEA) son las variaciones existentes en cuanto al modo

3. LOGSE. Título IV: De la calidad de la enseñanza. Art. 55. Los Poderes públicos prestarán una atención prioritaria al conjunto de factores que favorecen la calidad y mejora de la enseñanza, en especial a: a) La cualificación y formación del profesorado. b) La programación docente. c) Los recursos educativos y la función directiva. d) La innovación y la investigación educativa. e) La orientación educativa y profesional. f) La inspección educativa. g) La evaluación del sistema educativo. 


\section{TABLA 3: Factores Influyentes en el desempeño profesional del orientador.}

\begin{tabular}{|c|c|}
\hline \multicolumn{2}{|c|}{$\begin{array}{l}\text { DESEMPEÑO PROFESIONAL DE LOS ORIENTADORES } \\
\text { FACTORES INFLUYENTES }\end{array}$} \\
\hline DIRECTOS & INDIRECTOS \\
\hline $\begin{array}{l}\text { 1. Apoyo Institucional } \\
\text { - ¿Apoyo a la LOGSE? } \\
\text { - ¿Apoyo a la ORIENTACIÓN? }\end{array}$ & $\begin{array}{l}\text { 3. Experiencia previa } \\
\text { - Psicólogo } \\
\text { - Pedagogo } \\
\text { - Profesor - Primaria } \\
\text { - Profesor - Secundaria } \\
\text { - Orientador } \\
\text { 4. Prevalencia personal hacia } \\
\text { - La Tarea } \\
\text { - La Relación profesional } \\
\text { - Tarea = Relación } \\
\text { 5. Profesores del centro } \\
\text { - ¿Apoyan la LOGSE? } \\
\text { - ¿Apoyan la Orientación? } \\
\text { - Tipo dotación Dpto. Orientación } \\
\text { 6. Tipo de acceso al puesto } \\
\text { - Méritos } \\
\text { - Oposición } \\
\text { 7. Formación previa } \\
\text { - Psicólogo } \\
\text { - Pedagogo }\end{array}$ \\
\hline
\end{tabular}

de funcionamiento interno de los Dptos. de Orientación. Pero ¿qué está motivando dichas variaciones? Parece ser que, por un lado, son las diferencias entre centros respecto a los profesionales adscritos a los Dptos. de Orientación, con la consiguiente falta de recursos humanos para la atención a la diversidad en muchos de ellos, y por otro lado, la falta de unificación de criterios entre todos los Dptos. de orientación respecto a cómo deben realizarse determinadas actuaciones.

Por último añadimos otras opiniones y conclusiones extraídas durante la realización del presente estudio, y que corroboran en gran parte los datos aparecidos en estudios precedentes sobre la orientación y los orientadores en centros de educación secundaria públicos (Martín, 99; Documento de los orientadores de IESs y CEAs de la Subdirección Territorial Madrid-Centro).

Estos comentarios, vertidos fundamentalmente en las entrevistas y el registro son el reflejo de las opiniones más reiteradas de los orientadores de la muestra, las cuales se encuentran en estrecha relación con los datos obtenidos en los esquemas. Si bien podemos considerar estos instrumentos como más objetivos por el tipo de preguntas y aquéllos como más subjetivos por tratarse de un tipo de preguntas más abiertas y más proclives a extraer las 
preocupaciones y sentimientos personales que les invaden, podemos apreciar que unos datos complementan a los otros. Sirva como ejemplo la preocupación manifestada por muchos orientadores en la entrevista y el registro cuando comentan que se les asigna impartir materias que no son de su especialidad en vez de la asignatura Psicología, y después en los esquemas comprobamos que la mitad de los orientadores imparte esta materia y la otra mitad cualquier otra que se le asigne. Otra cuestión que plantean los orientadores en la entrevista es la multiplicidad de funciones que deben realizar que a su vez les impide llevar a cabo las funciones principales con la profundidad que desearían, y como podemos ver en los esquemas la cantidad de tareas y funciones es bastante amplia. Después de estas afirmaciones adquiere sentido su preocupación por no ser capaces de llevar a cabo una Atención a la Diversidad más extensa y profunda (ver ANEXO VI).

Creemos que el estudio de lo que realmente hacen los orientadores en su práctica cotidiana a través de los «esquemas de trabajo» propuestos, nos puede llevar a entender mejor el complejo entramado de variables que están influyendo para que ésta se desarrolle de una forma más o menos satisfactoria para todos los agentes educativos implicados, y en último término para los principales protagonistas y beneficiarios de la orientación educativa que son los alumnos. A pesar de que vemos este tipo de aproximación tan útil como prometedora, consideramos que es necesaria más investigación tanto contextual como teórica sobre este campo, para dar más luz a la definición de un puesto de trabajo tan complejo como apasionante que es el de orientador educativo.

\section{Propuesta de trabajo}

Tratando de acotar todos los factores que están influyendo en el desempeño real y cotidiano de cada orientador en su centro, se propone un modelo orientativo de siete variables que consideramos importantes no sólo por lo inevitable de su aparición, sino por la profundidad de su incidencia según se orienten hacia un polo u otro. Dichos factores los hemos extraído sintetizando todos los datos del estudio, tanto los esquemas y registros como las entrevistas semiestructuradas y los hemos dividido en directos e indirectos según la naturaleza y profundidad de su influencia.

Con ello pretendemos facilitar una visión global de algunos de los muchos factores que hemos encontrado, pero que por supuesto no agota cualquier otro tipo de aproximación al puesto de orientador.

\section{Referencias bibliográficas}

Alonso Tapia, J. (1995). Orientación educativa. Teoría, evaluación e intervención. Madrid: Síntesis.

Bassedas, E., Marrodan, T., Oliván, M., Planas, M., Rossell, M., Seguer, M., Vilella, M. (1991). Intervención educativa y diagnóstico psicopedagógico. Barcelona: Paidós

Hortelano, M. A. y Pascual, J .J. (1997). El departamento de Orientación. Salamanca: Amarú.

Martín, E. y Solé, I. (1990). Intervención psicopedagógica y actividad docente: claves para una colaboración necesaria. En Coll, C., Palacios, A., Marchesi, A (comps.). Desarrollo psicológico y educación, II. Psicología de la educación. Madrid: Alianza. 
Martín, E. La intervención psicopedagógica en el ámbito de gestión del MEC: la historia de un difícil pero imprescindible factor de calidad. (1999). Infancia y Aprendizaje, $\mathrm{n}^{\circ} 87$.

Orientadores de IESs y CEAs de la Subdirección Territorial Madrid-Centro (1999). El Dpto. de Orientación en los IESs y en los CEAs: Análisis, valoración y propuestas de mejora del modelo actual de organización y funcionamiento. Madrid: Documento interno.

Sánchez, E. y Ochoa de Alda, I. (1995). Profesores y psicopedagogos: propuesta para una relación compleja. Aula de innovación educativa, 38, 67-79.

Sánchez, E.: El asesoramiento psicopedagógico: un estudio observacional sobre las dificultades de los psicopedagogos para trabajar con los profesores. Infancia y Aprendizaje. (en prensa).

Santana Vega, L. E. (1993). Los Dilemas en la Orientación Educativa. Buenos Aires, Argentina: Cincel.

Selvini Palazolli, M. (1986). El mago sin magia. Madrid: Paidós. (original: Il mago smagato, 1976, Milán: Feltrinelli).

Solé, I. y Colomina, R (edits) (1999). La intervención psicopedagógica en centros educativos. Un espacio profesional polémico. Infancia y Aprendizaje, nº 87, monográfico.

Solé, I. (1998). Orientación Educativa e Intervención Psicopedagógica. Barcelona: Horsori-ICE.

VV.AA (1999). Actas de las II Jornadas de «Psicología de la Educación» en Castilla y León. León: COP de Castilla y León.

\section{Legislación educativa de referencia}

Ley Orgánica 1/1990 de 3 de Octubre de Ordenación General del Sistema Educativo (LOGSE)

Real Decreto 1345/1991, de 6 de sept. por el que se establece el Currículo de la Educación Secundaria Obligatoria.

Real Decreto 1007/1991, de 14 de junio, por el que se establecen las Enseñanzas Mínimas correspondientes a la ESO.

Real Decreto 1701/1991, de 29 de nov., por el que se establecen especialidades del cuerpo de profesores de Enseñanza Secundaria (Psicología y Pedagogía)

Real Decreto 83/1996, de 26 de enero, por el que se aprueba el Reglamento Orgánico de los Institutos de Educación Secundaria.

Resolución de 29 de abril de 1996, de la Dirección General de Centros Escolares, sobre organización de los Departamentos de Orientación en Institutos de Educación Secundaria.

Resolución de 30 de abril de 1996, de la Dirección General de Renovación Pedagógica por la que se dictan instrucciones sobre el Plan de Actividades de los Departamentos de Orientación de los Institutos de Educación secundaria. 


\section{ANEXO 1. \\ Registro}

1. ACTUALMENTE EN COLEGIO: PÚBLICO / PRIVADO / CONCERTADO

2. AÑOS DE EXPERIENCIA EN

- Orientador: PÚBLICA: / PRIVADA:

- Profesor: PRIMARIA: / SECUNDARIA:

3. FORMACIÓN: PSICOPEDAGOGO / PEDAGOGO / PSICÓLOGO

4. COMPONENTES DEL DPTO. DE ORIENTACIÓN:

5. PROFESORES DE APOYO (ESPECÍFICO) Y CUÁNTOS:

- AUDICIÓN Y LENGUAJE

- PEDAgogía TERAPÉUtica

- FISIOTERAPÉUTA

6. TIEMPO DEDICADO A CADA UNA DE LAS 3 FUNCIONES GENERALES (en porcentaje)

a) APOYO AL PROCESO DE ENSEÑANZA Y APRENDIZAJE

b) ORIENTACIÓN ACADÉMICA Y PROFESIONAL

c) APOYO A LA ACCIÓN TUTORIAL

7. POSEO FORMACIÓN ESPECÍFICA EN:

- TRABAJO EN EQUIPO / HABILIDADES DE NEGOCIACIÓN /

- DIRIGIR REUNIONES / ASESORAMIENTO COLABORATIVO

8. QUIÉN SOLICITA AYUDA /COLABORACIÓN HABITUALMENTE:

- TUTORES / PADRES / PROFESORES DE ÁMBITO

- ALUMNOS / DIRECTOR / PROFESORES DE ÁREA

9. CÓMO SE SOLICITAN LAS PETICIONES DE AYUDA/COLABORACIÓN Y QUIÉN:

- CON HOJA DE DEMANDA

- DE PALABRA Y PERSONALMENTE

- DE PALABRA EN REUNIONES DE EQUIPO

10. CUÁNDO SE SOLICITA AYUDA EN CASO DE PROBLEMA Y QUIÉN:

- AL VER INDICIOS DE PROBLEMA

- CUANDO EL PROBLEMA LLEVA BASTANTE TIEMPO

- CUANDO EL PROBLEMA DESBORDA AL PETICIONARIO

- ANTE SITUACIONES DE CRISIS

11. TIPO DE COLABORACIÓN HABITUAL Y CON QUIÉN

- COLABORACIÓN ACTIVA (REPARTO DE TAREAS)

- DICE QUE SÍ PERO LUEGO NO HACE NADA

- NO QUIERE COLABORAR 
12. TIPOS DE REUNIONES DEL DPTO. Y PERIODICIDAD DE LAS MISMAS

13. TIPOS DE REUNIONES DEL ORIENTADOR A LO LARGO DEL AÑO

14. ¿SE LLEVA A LA PRÁCTICA LO ACORDADO?

- SIEMPRE / HABITUALM. / A VECES / NUNCA

15. ¿SE NEGOCIAN / CONSENSÚAN LAS ACTUACIONES QUE HAY QUE LLEVAR A CABO? ¿CON QUIÉN?

16. ¿SE TRABAJA EN EQUIPO? ¿QUIÉNES?

17. DEDICACIÓN DE TIEMPO AL PRINCIPIO DE CURSO Y DURANTE CADA PROB. A

- DEFINIR EL CONTEXTO DE TRABAJO: Sí (PPIO CURSO/ EN CADA PROB) / NO

- CREAR UNA RELACIÓN DE COLABORACIÓN: SÍ (PPIO CURSO/ EN CADA PROB) / NO

- DEFINIR LOS PROBLEMAS CONJUNTAMENTE: SÍ (PPIO CURSO/ EN CADA PROB) / NO

- BUSCAR SOLUCIONES CONJUNTAMENTE: SÍ (PPIO CURSO/ EN CADA PROB) / NO

- REPARTIR TAREAS: SÍ (PPIO CURSO/ EN CADA PROB) / NO

- EVAluar los RESUltados CONJUNTAM.: Sí (PPIO CURSO/ EN CADA PROB) / NO

18. LA PERCEPCIÓN QUE TIENEN LOS DEMÁS PROFESIONALES DEL CENTRO SOBRE LO QUE ES UN ORIENTADOR ES: CORRECTA / INCORRECTA

19. ¿QUÉ ACTITUD HAY EN LOS CENTROS ESCOLARES HACIA EL ORIENTADOR?

- BUENA/MALA

- DE INDIFERENCIA/ DE COLABORACIÓN

20. CREO QUE DEBERÍA HABER MÁS FORMACIÓN/INFORMACIÓN EN LOS TEMAS SIGUIENTES:

- ORIENTADORES:

- PROFESORES: 


\section{ANEXO 2. \\ Entrevista}

A) FUNCIONES GENERALES

1. Apoyo a la Acción Tutorial

- qué-quién-cómo-cuándo-cuánto

- dificultades

- recursos

- proceso

2. Orientación Académica y Profesional

- qué-quién-cómo-cuándo-cuánto

- dificultades

- recursos

- proceso

3. Apoyo al proceso de Enseñanza y Aprendizaje

- qué-quién-cómo-cuándo-cuánto

- dificultades

- recursos

- proceso

B) OTRAS FUNCIONES

- Docencia

- Trabajo con las familias

- Relación con otros Dptos. del área

C) OTRAS CUESTIONES

- Tareas

- Modelo de Trabajo

- Colaboración con los demás Profesionales

- Ubicación en el Centro

- Cómo le ven

- Satisfacción Personal (Expectativas/Atribuciones)

- Un Futuro mejor. Cambios 


\section{ANEXO 3. \\ ESQUEMA I: Plan de Acción Tutorial (PAT)}

\begin{tabular}{|c|c|c|c|c|c|c|c|}
\hline \multirow{11}{*}{ PLANIFICACIÓN } & \multirow{4}{*}{ ELABORACIÓN } & \multirow{3}{*}{\multicolumn{2}{|c|}{ Documento inicial }} & \multicolumn{4}{|c|}{ Mismo que año anterior } \\
\hline & & & & \multirow{2}{*}{\multicolumn{4}{|c|}{$\begin{array}{l}\text { Año anterior y aportaciones de la evaluación } \\
\text { Nuevo }\end{array}$}} \\
\hline & & & & & & & \\
\hline & & \multicolumn{2}{|c|}{ Documento final } & \multicolumn{4}{|c|}{ Tutores / Orientador / Otros } \\
\hline & \multirow{3}{*}{$\begin{array}{l}\text { PRESENTACIÓNA } \\
\text { TUTORES }\end{array}$} & \multirow{2}{*}{ Sí } & \multirow{2}{*}{ Aportaciones } & \multirow{2}{*}{\multicolumn{4}{|c|}{\begin{tabular}{|l|} 
Sí \\
NO \\
\end{tabular}}} \\
\hline & & & & & & & \\
\hline & & \multicolumn{6}{|l|}{ NO } \\
\hline & \multirow{2}{*}{$\begin{array}{l}\text { PRESENTACIÓN A } \\
\text { LA CCP }\end{array}$} & Quién & & & Cómo & & \\
\hline & & \multicolumn{6}{|c|}{ Aportaciones (quién / qué) } \\
\hline & \multirow{2}{*}{$\begin{array}{l}\text { PRESENTACIÓN } \\
\text { AL CLAUSTRO }\end{array}$} & Quién & & & Cómo & & \\
\hline & & \multicolumn{6}{|c|}{ Aportaciones (quién / qué) } \\
\hline \multirow{2}{*}{\multicolumn{7}{|c|}{$\begin{array}{l}\text { ¿QUEDA CLARO PARA TODOS QUÉ SE VA A HACER, CÓMO Y QUIÉN, Y QUÉ, QUIÉN Y CÓMO SE VA A } \\
\text { EVALUAR? }\end{array}$}} & Sí \\
\hline & & & & & & & NO \\
\hline \multicolumn{7}{|c|}{ ¿LA DECISIÓN FINAL HA SIDO CONSENSUADA POR TODOS? } & $\begin{array}{ll}\text { SÍ } \\
\text { NO }\end{array}$ \\
\hline
\end{tabular}

\begin{tabular}{|c|c|c|c|}
\hline \multirow{8}{*}{ ACTUACIÓN } & $\begin{array}{l}\text { TIPOS DE ACTIVIDADES } \\
\text { DEL PAT }\end{array}$ & \multicolumn{2}{|c|}{$\begin{array}{l}\text { - Acogida, delegado, cohesión grupal, evaluación ens-aprje. } \\
\text { - Otras: }\end{array}$} \\
\hline & & \multicolumn{2}{|r|}{$\mathrm{M}=$ material $/ \mathrm{A}=$ asesoramiento $/ \mathrm{P}=$ presencialmente } \\
\hline & $\begin{array}{l}\text { TIPOS DE COLABORACIÓN } \\
\text { EN LAS ACTIVIDADES } \\
\text { (señalar: M, A o P) }\end{array}$ & \multicolumn{2}{|c|}{ 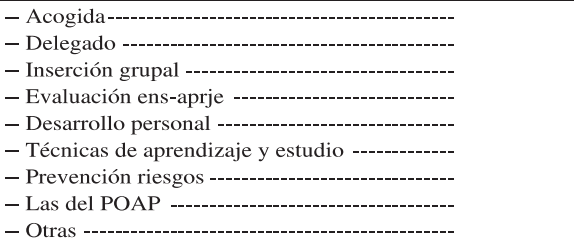 } \\
\hline & \multirow{3}{*}{\multicolumn{2}{|c|}{$\begin{array}{l}\text { CUÁNTOS TUTORES PIDEN } \\
\text { COLABORACIÓN (\%) }\end{array}$}} & Material \\
\hline & & & Asesoramiento \\
\hline & & & Presencialmente \\
\hline & \multicolumn{2}{|l|}{$\begin{array}{l}\text { OTRAS COLABORACIONES EN } \\
\text { LA H. DE TUTORÍA }\end{array}$} & $\begin{array}{l}\text { - Alumnos de prácticas } \\
\text { - ONGs }\end{array}$ \\
\hline & \multicolumn{2}{|l|}{ HORARIO TUTORÍA } & $-1^{\mathrm{a}}$ hora / intermedia / última / última y viernes \\
\hline
\end{tabular}

\begin{tabular}{|c|c|c|}
\hline \multirow{6}{*}{ SUPERVISIÓN } & DECISIÓN DE REUNIRSE & $\begin{array}{l}\text { Consensuada por todos } \\
\text { Consensuada por parte }(\%) \\
\text { Decide jefatura de estudios }\end{array}$ \\
\hline & FRECUENCIA REUNIONES & $\begin{array}{l}\text { - Semanal / Quincenal / Mensual / Trimestral / } 2 \text { al año / } \\
1 \text { al año }\end{array}$ \\
\hline & ASISTENCIA REUNIONES & $\begin{array}{l}\text { - Jefe de Estudios } \\
\text { - Tutores (cuántos) } \\
\text { - Orientador } \\
\text { - Otros } \\
\end{array}$ \\
\hline & PRESIDE LA REUNIÓN & - Jefe estudios / Orientador / Tutor / Otro \\
\hline & HORA DE LA REUNIÓN & - Intermedia / Última / Última y viernes \\
\hline & QUÉ SE SUPERVISA & Desarrollo de la actividad / planificar la siguiente /Otras \\
\hline
\end{tabular}

\begin{tabular}{|l|l|l|}
\hline \multirow{5}{*}{ EUÁNDO } & $\begin{array}{l}\text { - Junio / Septiembre } \\
\text { - No se hace }\end{array}$ \\
\hline \multirow{3}{*}{ QUIÉN } & $\begin{array}{l}\text { - Jefe de estudios } \\
\text { - Orientador } \\
\text { - Tutores } \\
\text { - Otros (ámbito, apoyo, FOL...) }\end{array}$ \\
\cline { 2 - 3 } & $\begin{array}{l}\text { - Memoria de actividades } \\
\text { - Especificación objetivos, metodología, duración actividades, secuenciación } \\
\text { - Entrevista con el orientador } \\
\text { - Reunión final de evaluación }\end{array}$ \\
\hline \multirow{2}{*}{ QUÉ / CÓMO } & $\begin{array}{l}\text { ¿Qué se incorpora al } \\
\text { año siguiente? }\end{array}$ \\
\hline
\end{tabular}




\section{ANEXO 4.}

\section{ESQUEMA II: Plan de Orientación Académica y Profesional (POAP)}

\begin{tabular}{|c|c|c|c|c|c|c|c|}
\hline \multirow{13}{*}{ PLANIFICACIÓN } & \multirow{4}{*}{ ELABORACIÓN } & \multirow{3}{*}{\multicolumn{2}{|c|}{ Documento inici }} & \multicolumn{4}{|c|}{ Mismo que año anterior } \\
\hline & & & & \multicolumn{4}{|c|}{ Año anterior y aportaciones de la evaluación } \\
\hline & & & & \multicolumn{4}{|c|}{ Nuevo } \\
\hline & & \multicolumn{2}{|c|}{ Documento final } & \multicolumn{4}{|c|}{ Tutores / Orientador / FOL / Otros } \\
\hline & \multirow{3}{*}{$\begin{array}{l}\text { PRESENTACIÓN } \\
\text { A TUTORES }\end{array}$} & \multirow{2}{*}{ Sí } & \multirow{2}{*}{\multicolumn{2}{|c|}{ Aportaciones }} & \multicolumn{3}{|c|}{ Sí } \\
\hline & & & & & \multicolumn{3}{|c|}{$\mathrm{NO}$} \\
\hline & & NO & & & & & \\
\hline & \multirow[b]{2}{*}{$\begin{array}{l}\text { PRESENTACIÓN } \\
\text { A LA CCP }\end{array}$} & \multicolumn{2}{|c|}{ Quién } & & & \multicolumn{2}{|l|}{ Cómo } \\
\hline & & \multicolumn{2}{|c|}{$\begin{array}{l}\text { Aportaciones } \\
\text { (Quién / Qué) }\end{array}$} & & & & \\
\hline & \multirow[b]{2}{*}{$\begin{array}{l}\text { PRESENTACIÓN } \\
\text { AL CLAUSTRO }\end{array}$} & \multicolumn{2}{|c|}{ Quién } & & & \multicolumn{2}{|l|}{ Cómo } \\
\hline & & \multicolumn{2}{|c|}{$\begin{array}{l}\text { Aportaciones } \\
\text { (Quién / Qué) }\end{array}$} & & & & \\
\hline & \multirow{2}{*}{\multicolumn{6}{|c|}{$\begin{array}{l}\text { ¿QUEDA CLARO PARA TODOS QUÉ SE VA A HACER, CÓMO Y QUIÉN, Y } \\
\text { QUÉ, QUIÉN Y CÓMO SE VA A EVALUAR? }\end{array}$}} & SÍ \\
\hline & & & & & & & $\mathrm{NO}$ \\
\hline
\end{tabular}

\begin{tabular}{|c|c|c|}
\hline \multirow{7}{*}{ ACTUACIÓN } & $\begin{array}{l}\text { TIPOS DE ACTIVIDADES } \\
\text { DEL POAP }\end{array}$ & $\begin{array}{l}\text { - Programaciones Didácticas } \\
\text { - Dentro de la hora de tutoría } \\
\text { - Tutorías individuales: alumnos / familias } \\
\text { - Reuniones de padres }\end{array}$ \\
\hline & CUÁNDO & $\begin{array}{l}\text { - Toda la ESO } \\
\text { - Sólo en algún curso: } 1^{\circ} / 2^{\circ} / 3^{\circ} / 4^{\circ} / 1^{\circ} \mathrm{B} / 2^{\circ} \mathrm{B} \\
\text { - Trimestre: } 1^{\circ} / 2^{\circ} / 3^{\circ}\end{array}$ \\
\hline & \multirow[b]{2}{*}{$\begin{array}{l}\text { TIPOS DE } \\
\text { COLABORACIÓN EN } \\
\text { LAS ACTIVIDADES } \\
\text { (señalar: M, A o P) }\end{array}$} & $\mathrm{M}=$ material $/ \mathrm{A}=$ asesoramiento $/ \mathrm{P}=$ presencialmente \\
\hline & & 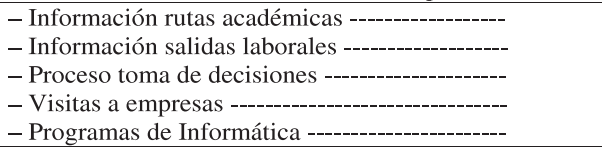 \\
\hline & \multirow{3}{*}{$\begin{array}{l}\text { CUÁNTOS TUTORES } \\
\text { PIDEN COLABORACIÓN } \\
(\%)\end{array}$} & material \\
\hline & & asesoramiento \\
\hline & & presencialmente \\
\hline
\end{tabular}

\begin{tabular}{|l|l|l|}
\hline \multirow{2}{*}{ SUPERVISIÓN } & QUIÉN & $\begin{array}{l}\text { - Jefe de Estudios } \\
- \text { Tutores (cuantos) } \\
- \text { Orientador } \\
- \text { Otros }\end{array}$ \\
\cline { 2 - 3 } & FRECUENCIA & Mensual / Trimestral / Anual / Otra \\
\cline { 2 - 3 } & QUÉ & Desarrollo de actividades / Planificar las siguientes / Otras \\
\hline
\end{tabular}

\begin{tabular}{|l|l|l|}
\hline \multirow{2}{*}{ EVALUACIÓN } & CUÁNDO & $\begin{array}{l}\text { - Junio / Septiembre } \\
\text { - No se hace }\end{array}$ \\
\cline { 2 - 3 } QUIÉN & $\begin{array}{l}\text { - Jefe de estudios } \\
\text { - Orientador } \\
\text { - Tutores } \\
\text { - Otros (ámbito, apoyo, FOL) }\end{array}$ \\
\cline { 2 - 3 } QUÉ / CÓMO & $\begin{array}{l}\text { - Memoria de actividades } \\
\text { - Especificación objetivos, metodología, duración actividades, } \\
\text { secuenciación } \\
\text { - Entrevista con el orientador } \\
\text { - Reunión final de evaluación }\end{array}$ \\
\cline { 2 - 3 } & TRASCENDENCIA & $\begin{array}{l}\text { ¿Qué se incorpora } \\
\text { al año siguiente? }\end{array}$ \\
\hline
\end{tabular}




\section{ANEXO 5. \\ ESQUEMA III: Proceso de Enseñanza y Aprendizaje (PEA)}

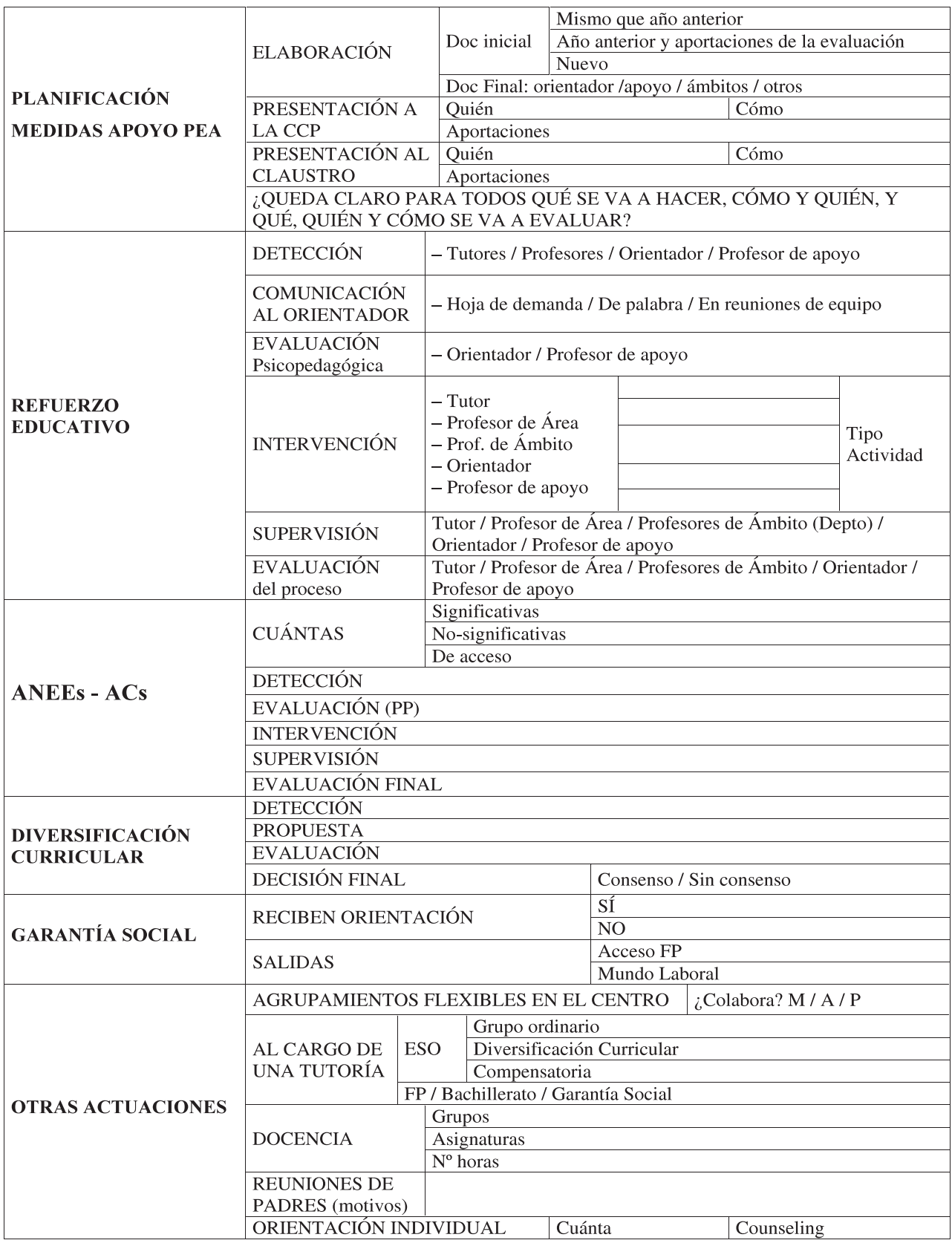




\section{ANEXO VI. \\ Principales preocupaciones personales-profesionales manifestadas por los Orientadores}

- Gran multiplicidad de funciones asignadas al Dpto. de Orientación en general, y al orientador en particular (PAT, POAP, PEA, Docencia, CCP, Claustro, Evaluaciones, Reuniones de padres, Asesoramiento individual a padres y alumnos, Conexión con EOEPs, etc.).

- Asignación de demasiadas horas docencia que restan tiempo a la orientación, p. ej. orientadores que en centros de 1000 alumnos imparten 8 ó 9 horas.

- Asignación de asignaturas que no son de la especialidad, p. ej. Religión, Alternativa al estudio, Ética, Procesos de Comunicación, Transición a la Vida Adulta y Activa, etc., con la exigencia añadida de preparar materias que no se dominan, lo cual resta tiempo para la orientación educativa ${ }^{4}$.

- Apropiación de la materia «Psicología», que es la más propia de la especialidad del profesor de «Psicología y Pedagogía», por profesores de otra especialidad por el hecho de no estar considerado el Dpto. de Orientación como departamento didáctico, cometiéndose una clara ambigüedad e injusticia, puesto que en la realidad sí que imparten docencia. En estos casos, el profesor que reclama esa asignatura, suele rechazar impartir otras materias que sí son de su especialidad.

- Falta de dotación de personal en los Dptos. de Orientación que conlleva más trabajo para los que están y provoca una insuficiente atención a los temas de la Atención a la Diversidad.

- Falta de unificación de criterios entre los orientadores sobre cómo se deben llevar a cabo determinadas actuaciones, especialmente las relacionadas con refuerzo educativo, Adaptaciones Curriculares y atención a ANEEs.

- Invisibilidad de las tareas de orientación que provoca críticas y rechazos injustificados por parte de otros agentes educativos.

- Falta de paridad en la ratio número de alumnos por orientador (y por miembros del Dpto. de Orientación), es decir, que en todos los centros hay un único orientador, tenga éste 200 ó 2000 alumnos, siendo esto un caso único entre todos los profesores de Secundaria.

- «Abandono cultural» por parte del MEC (actualmente Dpto. de Educación y Cultura), con respecto a actualización, formación continua y unificación de criterios de trabajo.

- Falta de una definición clara de las funciones del orientador al comienzo del curso y al abordar cada problema en concreto.

4. «Orden de 29 de junio de 1994 por la que se aprueban las instrucciones que regulan la Organización y Funcionamiento de los IES». B) Profesores del Dpto. de Orientación. 83. a) Entre 6 y 9 períodos lectivos, dedicados a impartir materias optativas relacionadas con su especialidad y a la atención de grupos de alumnos que sigan programas específicos, según determine la jefatura de estudios. 